\title{
Quelques méthodes de choix des génotypes à partir d'essais multilocaux
}

\author{
Patrick VINCOURT ( ${ }^{1}$ ), Maurice DERIEUX* \& André GALLAIS** \\ I.N.R.A., Station d'Amélioration des Plantes Fourragères, F 86600 Lusignan \\ * I.N.R.A., Station d'Amélioration des Plantes, Mons-en-Chaussée, F 80200 Péronne \\ ** GIS-Moulon, F 91190 Gif-sur-Yvette
}

RÉSUMÉ

On montre, sur des exemples, comment trois méthodes d'analyse des données provenant d'essais multilocaux, dérivées en particulier des travaux de MANDEl (1971), BURdon (1979), GofFinet \& VincourT (1980), permettent d'enrichir la connaissance des sélectionneurs. Les effets des génotypes sont regardés comme fixés ou comme aléatoires. Ces techniques, qui permettent d'établir une continuité entre les concepts utilisés en sélection (héritabilité) et les critères empiriques de choix, peuvent être diffusées auprès des sélectionneurs au travers des programmes Hender et MUltiloc (développés en Fortran sur Mini-6, CII-HB).

Mots clés additionnels : Interaction génotype $\times$ lieu, adaptation, index de sélection, coefficient de détermination génétique.

How to select genotypes from multilocational data.

From the approaches of MANDEL (1971), BURDON (1979), and GofFinET \& VINCOURT (1980), three methods of analysis of genotype $x$ environment data are presented through experimental results. Estimations obtained for genotype performance, with fixed or random effects, seemed to be able to improve the value of selection criteria, by integrating in the same approach both empirical practice and the concepts that are commonly used in selection, such as heritability or predicted gain.

Additional key words : Genotype $\times$ environment interaction, adaptation, selection indices, coefficient of genetic determination.

\section{INTRODUCTION}

S'il veut conférer au matériel génétique une adaptation géographique suffisamment large, le sélectionneur est conduit, selon l'étape au cours du cycle de sélection, soit à tester un grand nombre de familles dans 2 ou 3 lieux, soit à apprécier la valeur de produits quasi finis dans un réseau d'essais plus important. Le problème se pose alors, d'une part, de condenser l'information provenant de ces essais multilocaux afin de prendre des décisions sur les génotypes, d'autre part, de porter un jugement sur les lieux de test afin éventuellement d'alléger le dispositif mis en place ultérieurement.

Les règles de décisions courantes s'inscrivent le plus souvent dans le cadre du modèle à effets fixés (YATES \& COCHRAN, 1938 ; FINLAY \& WILKINSON, 1963 ; MANDEL, 1971 ; DENIS, 1979, 1980). Il est parfois fait

(1) Adresse actuelle : INRA, Station d'Amélioration des Plantes, Centre de Recherches de Montpellier, Domaine de Melgueil, 34130 Mauguio. référence à des composantes de la variance (EBERHART \& RUSSEL, 1966 ; UTZ, 1972), mais il s'agit en fait d'une décomposition de certains carrés moyens pratiquée à partir de modèles dont toutes les hypothèses n'ont pas été explicitées, en particulier l'absence de corrélations entre valeur génétique moyenne et paramètre de stabilité (HARDWICK, 1981).

Ces techniques présentent en outre 2 inconvénients majeurs à nos yeux :

- On ne dispose généralement pas du même niveau de précision dans les différents lieux. Or la qualité d'un lieu de sélection dépend à la fois de son pouvoir de prédiction - c'est-à-dire de son aptitude à donner des génotypes un classement fiable sur une aire géographique suffisamment vaste - et de son pouvoir de révélation de certaines sensibilités. Ces 2 caractéristiques ne sont pas obligatoirement liées et la précision expérimentale, regardée non pas seulement comme un outil statistique mais comme un critère agronomique, intervient pour une part non négligeable dans la définition du profil d'un lieu de test. 
- Adopter le modèle à effets fixés revient à passer sous le silence le fait que la gamme de variation génétique attendue n'est en général pas très grande (variance) et que l'on s'attend a priori à trouver des liaisons entre les comportements dans les différents lieux (corrélation).

Les 3 modèles présentés dans cet article à partir d'exemples répondent à des situations et à des objectifs différents et permettent de pallier tout ou partie de ces inconvénients.

\section{MATÉRIEL ET MÉTHODES}

\section{A. Les premières étapes de sélection}

Au cours des premières étapes de la sélection, le tri est effectué parmi un grand nombre de génotypes avec une intensité de sélection généralement faible. Le souci principal est alors d'apprécier la valeur d'adaptation générale du matériel. Le recours aux tests multilocaux permet, d'une part, d'accroître la précision de cette appréciation, d'autre part, de porter un jugement global sur la plasticité de la population soumise à la sélection et sur le système de test employé.

Deux voies d'élaboration du critère de sélection peuvent être envisagées.

\section{Approche multicaractère}

La démarche consistant à considérer les réalisations d'un caractère dans différents lieux comme des caractères différents avait été proposée par FALCONER (1960).

\section{a) Modèle}

L'analyse des données multilocales par une méthode analogue à celle des indices de sélection est utilisée notamment en amélioration forestière (BURDON, 1979). Le modèle correspondant à cette technique est le suivant :

$$
y_{i, 1}^{(j, k)}=m^{(j . k)}+G_{i}^{(j, k)}+E_{i, i}^{(j . k)}
$$

$\mathrm{j}$ indice lieu $; \mathrm{l}<\mathrm{j}<\mathrm{J} ; \mathrm{k}$ indice caractère, $1<\mathrm{k}<\mathrm{K}$

(0) $\mathrm{i}$ indice génotype, $1<\mathrm{i}<\mathrm{I}$; 1 indice répétition, hiérarchisé à l'indice lieu

$$
\text { le vecteur }\left(\begin{array}{l}
G_{i}^{(1,1)} \\
\vdots \\
G_{i}^{(J, K)}
\end{array}\right) \begin{aligned}
& \text { suit une loi normale cen- } \\
& \text { trée, de matrice de variance- } \\
& \text { covariance } \Sigma_{\mathrm{G}} \text { pour tout }{ }_{\mathrm{i}}
\end{aligned}
$$

suit une loi normale centrée de matrice de variancele vecteur $\left(\begin{array}{l}\mathrm{E}_{\mathrm{i}, \mathrm{i}}^{(1,1)} \\ \vdots \\ \mathrm{E}_{\mathrm{i} . \mathrm{i}}^{(J, \mathrm{~K})}\end{array}\right)$ covariance $\Sigma_{\mathrm{E}}$ diagonale par bloc, les covariances entre effets résiduels dans deux lieux différents étant nulles.

Comme dans le cas de la sélection multicaractère, le calcul de critères tels que le gain attendu en sélection et le coefficient de détermination génétique est possible et permet d'interpréter l'influence du choix des lieux sur l'efficacité de la sélection. Pour plus de détails, nous renvoyons le lecteur à un article précédent (VINCOURT \& GALLAIS, 1983).

\section{b) Données expérimentales}

Lors de la première étape du cycle de sélection récurrente du maïs ensilage (GALLAIS et al., 1983), les descendances des plantes $S_{0}$ croisées au testeur apparenté au groupe réciproque sont installées en 2 lieux : Lusignan (systématique) et l'un des lieux Rennes, La Minière, Dijon, Le Pin. Chaque lieu est représenté par 2 répétitions. Les matrices de variances-covariances génétiques $\left(\Sigma_{\mathrm{G}}\right)$ et résiduelles $\left(\Sigma_{\mathrm{E}}\right)$ sont obtenues par l'analyse de variance dans chaque lieu considéré comme un groupe de caractères. La covariance génétique entre lieux est calculée comme la covariance empirique entre moyennes phénotypiques.

A titre d'exemple, le tableau 1 donne les matrices $\Sigma_{\mathrm{G}}$ et $\Sigma_{\mathrm{E}}$ obtenues pour une synthétique testée en 1980 dans les lieux Lusignan et Rennes pour une part des descendances, Lusignan et Le Pin pour les autres. L'analyse porte sur 150 descendances groupées en 4 essais. Seules les variables « Rendement en matière sèche plante entière » (RDT) et «Teneur en matière sèche » (p. $100 \mathrm{MS}$ ) sont considérées ici.

\section{Modèle multiplicatif mixte}

\section{a) Modèle}

La modélisation de l'interaction génotype $\times$ milieu a été récemment analysée par MALLARD et al., 1983. Afin de synthétiser l'information sur les génotypes, la méthode d'analyse développée par MANDEL (1971) peut être adaptée à une situation où l'effet génotype est considéré comme alétoire (GOFFINET \& VINCOURT, 1980) :

$$
\mid \begin{aligned}
& Y_{i j l}=m+b_{j}+G_{i}+c_{j} D_{i}+R_{i j}+E_{i j} \\
& i \text { indice génotype }: l<i<I \\
& j \text { indice lieu }: 1<j<J \\
& 1 \text { indice répétition dans lieu }: 1<1 L_{j} \\
& b_{j} \text { et } c_{j} \text { sont des effets du milieu : } \\
& \quad \sum_{j} b_{j}=0 ; \quad \sum_{j} c_{j}=0 ; \quad \sum_{j} c_{J}^{2}=1
\end{aligned}
$$

(1) le couple $\left(G_{i}, D_{i}\right)$ suit une loi binormale d'espérance $(0,0)$ et de matrice de variance-covariance :

$$
\left(\begin{array}{c:c}
\sigma_{\mathrm{G}}^{2} & \rho \sigma_{\mathrm{G}} \sigma_{\mathrm{D}} \\
\hdashline \rho \sigma_{\mathrm{G}} \sigma_{\mathrm{D}} & \sigma_{\mathrm{D}}^{2}
\end{array}\right)
$$

$\mathrm{R}_{\mathrm{ij}}$ a une loi normale $\left(0, \sigma_{\mathrm{R}}^{2}\right)$

$E_{i j 1}$ a une loi normale $\left(0, \sigma_{(j) E}^{2}\right)$

Les aléatoires $\left(G_{i}, D_{i}\right), R_{i j}, E_{i j l}$ sont indépendantes.

Ce modèle conduit à définir pour chaque génotype 2 caractéristiques qui peuvent servir de base à un jugement sur la valeur générale et sur l'adaptation spécifique à un type de milieu.

Cette valeur générale n'est mesurée par $\widehat{G}_{i}$ que si, à chacun des lieux, peut être affecté le même poids pour représenter la zone à laquelle est destiné le génotype sélectionné. Dans le cas contraire, on triera les génotypes sur un index $\mathrm{G}_{\mathrm{i}}+\gamma \mathrm{D}_{\mathrm{i}}$. 
Remarque : En adoptant la démarche proposée par SCHEFFE (1959), on pourrait également prendre en compte les poids $\mathrm{p} \mathrm{j}$ des lieux au niveau des conditions supplémentaires du modèle (1) :

$$
\sum_{j} b_{j} p_{j}=0 ; \quad \sum_{j} c_{j} p_{j}=0
$$

Le critère $\hat{G}_{i}$ fournirait alors directement l'estimation de valeur générale et $\hat{\mathrm{D}}_{\mathrm{i}}$ mesurerait la stabilité de comportement vis-à-vis des variations de milieu présentes au sein de la zone cible.

Les estimations des $G_{i}$ et $D_{i}$ sont obtenues par régression linéaire sur les variables $Y_{\mathrm{ijl}}$ centrées et s'écrivent

$$
\begin{aligned}
& \hat{G}_{i}=\sum_{j} \alpha_{j}\left(Y_{i j}-Y_{. j}\right) \\
& \hat{D}_{i}=\sum_{j} \beta_{j}\left(Y_{i j .}-Y_{. j .}\right)
\end{aligned}
$$

Les coefficients $\alpha_{j}$ et $\beta_{j}$ dépendent de $\sigma_{G}^{2}, \sigma_{D}^{2}, \sigma_{R}^{2}$, $\sigma_{\mathrm{j}}^{2} \mathrm{E}$, et des $\left\{\mathrm{c}_{\mathrm{j}}\right\}$, qui peuvent être estimés à partir des données $\left\{Y_{\mathrm{ij}}\right\}$ selon cune procédure donnée en annexe.

\section{b) Données expérimentales}

Cette méthode est présentée à partir d'un essai multilocal de maïs-grain, conduit en 1978 pour la Station d'Amélioration des Plantes de Mons-en-Chaussée (M. DERIEUX). Ces données ont par ailleurs fait l'objet d'un traitement par l'approche multicaractère (VINCOURT \& GALlais, 1983).

\section{B. Le choix des variétés}

\section{Modèle}

Supposons que nous ayons à choisir, parmi un relativement petit nombre de génotypes (10 à 20), ceux qui présentent une bonne valeur générale ou un profil particulier dans certains lieux. Ce peut être le cas de formules commerciales sur lesquelles on possède déjà certaines références et qu'on souhaite tester sur un réseau plus large.

Dans cette situation, chaque génotype est, au moins en partie, connu individuellement et il convient d'adopter le modèle à effets fixés suivant :

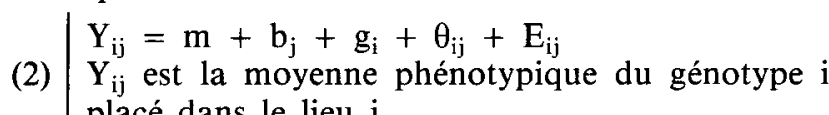
placé dans le lieu $\mathrm{j}$,

$\mathrm{m}, \mathrm{b}_{\mathrm{j}}, \mathrm{g}_{\mathrm{i}}, \theta_{\mathrm{ij}}$ sont des effets fixés

$\mathrm{g}_{\mathrm{j}}$ effet du génotype $\mathrm{i}$

$b_{j}$ effet du milieu $j$

$\theta_{\mathrm{ij}}$ interaction (génotype, lieu)

$E_{i j}$ est un effet aléatoire que nous supposerons de distribution normale centrée et de variance $\sigma_{j}^{2}$, dont on possède une estimation $\hat{\sigma}_{j}^{2}$ par ailleurs (analyse intra lieu).

Différentes techniques d'analyse des interactions $\theta_{\mathrm{ij}}$ ont été proposées (DENIS \& VINCOURT, 1982). Généralement elles ne prennent pas en compte les différences de précisions des lieux de test - c'est-à-dire l'inégalité des $\sigma_{\mathrm{j}}^{2}$. Elles s'attachent davantage à décrire le tableau des $\theta_{\mathrm{ij}}$, et nous retiendrons dans la suite la description proposée par MANDEL (1971), dont la forme la plus simple est :

$$
\theta_{i j}=c_{j} d_{i}+r_{i j} \text {. }
$$

On peut résumer cette approche de la façon suivante : on cherche des échelles des lieux $\left(c_{j}\right)$ et des génotypes $\left(d_{i}\right)$ qui conjointement produisent une approximation satisfaisante de l'interaction $\theta_{\mathrm{ij}}$.

Dans un certain nombre de cas, on connaît avec une bonne précision les valeurs des différents $\sigma_{\mathrm{j}}^{2}$. Nous proposons donc de substituer au modèle (2) le modèle suivant :

$$
\frac{Y i j}{\hat{\sigma}_{j}}=m^{\prime}+g_{i}^{\prime}+c_{j}^{\prime} \cdot d_{i}^{\prime}+r_{i j}+E_{i j}^{\prime}
$$

où $\hat{\sigma}_{\mathrm{j}}$ est l'estimation de l'écart-type intra-lieu.

L'avantage de ce modèle non orthodoxe est en particulier d'exhiber des paramètres ayant une signification assez concrète pour le décideur :

- $\mathbf{g}_{\mathbf{i}}^{\prime}$ est un paramètre d'adaptation générale qui est exprimé en unité d'écart-type intra-lieu : on s'affranchit ainsi de la liaison variance-moyenne provoquée par les écarts pédoclimatiques importants entre lieux et on dispose d'un paramètre sans dimension :

- $\mathrm{m}_{\mathrm{j}}^{\prime}$ est l'inverse du coefficient de variation (intralieu) cher aux expérimentateurs.

Si la variable $E_{i j}^{\prime}$ est distribuée normalement, la loi de $E_{i j}^{\prime}$ est celle d'un variable de STUDENT dont la convergence vers la loi normale centrée réduite est assurée dès que l'estimation $\hat{\sigma}_{j}^{2}$ repose sur un assez grand nombre de degrés de liberté (une centaine).

\section{RÉSULTATS ET DISCUSSION}

\section{A. Sélection en deux lieux pour le maïs ensilage}

L'examen du tableau 1 montre que, pour un même caractère - le rendement ou la teneur en matière sèche - les coefficients de corrélation génétique estimés entre lieux sont relativement élevés. La précision de ces estimations est bien entendu moindre que celle

\section{TABLEAU}

Sélection du maìs-ensilage : prise en compte des corrélations entre lieux.

Multilocational breeding for maize silage: taking in account the correlations between locations

\begin{tabular}{cccccccc}
\hline \hline \multirow{2}{*}{ Variables } & \multicolumn{2}{c}{ Lusignan } & \multicolumn{2}{c}{ Le Pin } & \multicolumn{2}{c}{ Rennes } \\
& $\begin{array}{c}\text { Rdt } \\
\text { (t/ha) }\end{array}$ & $\begin{array}{c}\text { \% ms } \\
\text { (point) }\end{array}$ & Rdt & $\%$ ms & Rdt & $\%$ ms \\
$\sigma_{\mathrm{G}}$ & 1,0 & 2,2 & 1,1 & 1,5 & 1,7 & 1,3 \\
$\sigma_{\mathrm{e}}$ & 1,1 & 1,8 & 1,1 & 1,4 & 1,8 & 1,4 \\
Corrélations & 1 & 0,170 & 0 & 0 & 0 & 0 \\
(a) & $-0,121$ & 1 & 0 & 0 & 0 & 0 \\
& $-0,756$ & $-0,532$ & 1 & 0,323 & 0 & 0 \\
& $-0,073$ & 0,700 & $-0,319$ & 1 & 0 & 0 \\
& 0,726 & 0,073 & (b) & (b) & 1 & 0,564 \\
& 0,009 & 0,094 & (b) & (b) & 0,161 & 1 \\
\hline \hline
\end{tabular}

(a) Corrélations génétiques sous la diagonale résiduelles au-dessus de la diagonale.

Genetic correlations below the diagonal and environmental correlations above the diagonal.

(b) Ces corrélations ne peuvent être estimées dans le dispositif utilisé.

These correlations cannot be estimated through this experimental design. 
que l'on aurait sur les corrélations phénotypiques. Toutefois, compte tenu du grand nombre de descendances testées, la valeur absolue de ces corrélations est toujours supérieure à 2 fois leur écart-type. On peut penser que les disparités de classement observées entre lieux sur la base des moyennes phénotypiques sont surtout dues à une héritabilité (au sens large) intra-lieu relativement faible.

Cette procédure de calcul permet d'estimer la valeur génétique attendue à Lusignan (resp. à Rennes) à partir des valeurs phénotypiques observées à Rennes comme à Lusignan. Ainsi :

$$
\begin{aligned}
& \mathrm{G}_{\mathrm{LL} \text {. RDT }}=0,512 \mathrm{P}_{\mathrm{LL} \text {. RDT }}-0,033 \mathrm{P}_{\mathrm{LLL} .{ }^{\circ} \mathrm{MIS}} \\
& +0,144 \mathrm{P}_{\mathrm{RE} . \mathrm{RDJ}}-0,020 \mathrm{P}_{\mathrm{RE} \% \mathrm{MS}} \\
& \mathrm{G}_{\text {Rt. RDT }}=0,304 \mathrm{P}_{\text {LU, RDT }}+0,063 \mathrm{P}_{\mathrm{LU} . \% \mathrm{Ms}} \\
& +0.599 \mathrm{P}_{\mathrm{RE.RDT}}-0,166 \mathrm{P}_{\mathrm{RH} \% \mathrm{MS}}
\end{aligned}
$$

Dans ces équations, les variables $\mathrm{P}$ représentent les écarts phénotypiques à la moyenne générale.

Le tableau 2 donne les coefficients de détermination génétique en fonction des lieux de test : il apparaît toujours avantageux de fusionner l'information provenant des 2 lieux. Il faut souligner que cette procédure n'enlève pas au sélectionneur l'initiative de choisir quels poids donner aux lieux, car le critère final de sélection se présente comme :

$$
I=a_{1} G_{\text {Lusignan }}+a_{2} G_{\text {Rennes }} \text {. }
$$

TABLEAU 2

Coefficients de détermination génétique en fonction du choix des lieux de test.

Coefficient of genetic determination according to the environment where the selection is worked out.

\begin{tabular}{lcccccc}
\hline \hline $\begin{array}{c}\text { Variables } \\
\text { prédictrices }\end{array}$ & \multicolumn{5}{c}{ Variables prédites } \\
& \multicolumn{1}{c}{ Lusignan } & \multicolumn{2}{c}{ Le Pin } & \multicolumn{2}{c}{ Rennes } \\
& Rdt & $\% 0 \mathrm{~ms}$ & Rdt & $\%$ ms & Rdt & $\%$ ms \\
Lusignan seul & 0,634 & 0,770 & 0,528 & 0,374 & 0,328 & 0,629 \\
Le Pin seul & 0,402 & 0,500 & 0,727 & 0,775 & - & - \\
Rennes seul & 0,371 & 0,611 & - & - & 0,699 & 0,658 \\
Lus + Le Pin & 0,699 & 0,809 & 0,783 & 0,795 & - & - \\
Lus + Rennes & 0,695 & 0,830 & - & - & 0,709 & 0,778 \\
\hline \hline
\end{tabular}

Pour raisonner le choix de ces coefficients $a_{i}$, il peut être utile d'examiner les réponses attendues en sélection directe ou indirecte, comme cela est fait dans la figure 1. Ainsi dans les essais conduits simultanément à Lusignan et au Pin, il semble préférable d'accorder un plus grand poids à la teneur en matière sèche évaluée au Pin et au rendement évalué à Lusignan. Pour les essais conduits à Lusignan et à Rennes, l'indice de sélection devrait prendre en compte surtout les valeurs obtenues à Rennes.

Notons enfin, que, si un même poids est affecté aux 2 lieux de test, le critère de sélection bâti selon cette procédure ne diffère pas sensiblement du critère empirique consistant à faire la moyenne arithmétique des performances obtenues dans les 2 lieux, au moins en ce qui concerne le classement des génotypes.

\section{B. Modèle mixte à effets génétiques aléatoires}

Les 8 lieux utilisés dans l'essai analysé selon cette méthode présentent une grande variation tant du

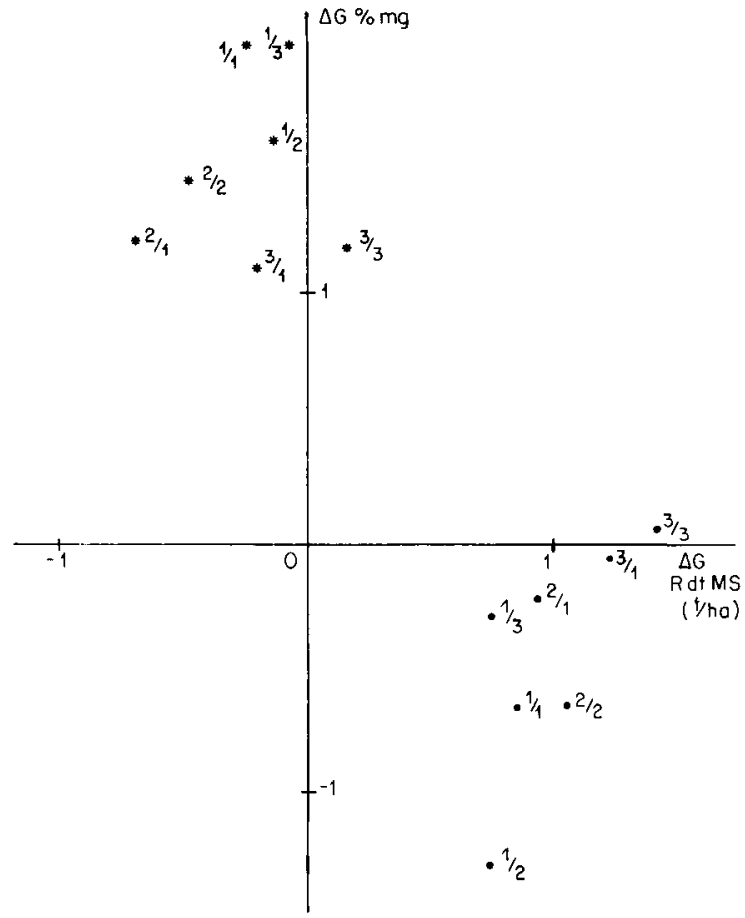

Figure 1

Gains espérés en sélection sur le Rdt (.) ou sur la teneur MS $(x)$ dans le lieu i à partir du lieu $j:(i / j)$.

Expected gain for yield (.) or dry matter content $(x)$ when the selection is worked out in the location y for an improvement in the location $i:(i / j)$.

Code lieux (locations) : 1 Lusignan, 2 Le Pin, 3 Rennes.

point de vue du niveau de production (tabl. 3 , paramètre $\mathrm{m}+\mathrm{b}_{\mathrm{j}}$ ) que de la précision intra-lieu (paramètre $\hat{\sigma}_{\mathrm{j}}$ ).

L'échelle définie par le paramètre $c_{j}$ permet de distinguer les génotypes qui sont davantage adaptés à Compiègne et Dieppe, ou au contraire à Rennes, Boigneville et Clermont-Ferrand.

Les estimations des paramètres génétiques, obtenus en utilisant la procédure donnée en annexe, sont les suivants :

$$
\begin{aligned}
\sigma_{\mathrm{G}}^{2} & =10,80 \\
\sigma_{\mathrm{D}}^{2} & =42,36 \\
\operatorname{Cov}(\mathrm{G}, \mathrm{D}) & =2,72 \\
\sigma_{\mathrm{R}}^{2} & =8,67
\end{aligned}
$$

TABLEAU 3

Caractérisation des lieux de test dans le modèle (1) (essai 12, Mons-en-Chaussée, 1978).

Description of the locations from the model (1).

\begin{tabular}{lccrr}
\hline \multicolumn{1}{c}{ Effets des lieux } & \multicolumn{1}{c}{} & & \\
& $\mathrm{m}+\mathrm{b}_{\mathrm{j}}$ & \multicolumn{1}{c}{$\mathrm{c}_{\mathrm{j}}$} & $\hat{\sigma}_{\mathrm{j}}$ & \multicolumn{1}{c}{$\mathrm{c}_{\mathrm{j}}^{\prime}$} \\
Compiègne & 66,8 & 0,707 & 2,85 & 0,648 \\
Rennes & 78,6 & $-0,467$ & 4,07 & $-0,347$ \\
La Minière & 70,1 & 0,170 & 4,37 & 0,293 \\
Verneuil l'Etang & 60,6 & $-0,053$ & 2,72 & 0,000 \\
Cld-Ferrand & 61,8 & $-0,281$ & 2,60 & $-0,405$ \\
Chateaudun & 76,3 & 0,044 & 3,91 & 0,062 \\
Dieppe & 53,8 & 0,226 & 2,88 & 0,170 \\
Boigneville & 74,5 & $-0,345$ & 3,38 & $-0,421$ \\
\hline
\end{tabular}


La variance résiduelle $\sigma_{\mathrm{R}}^{2}$ est inférieure ou égale aux variances résiduelles $\hat{\sigma}_{j}^{2}$ et largement inférieure à la variance $\sigma_{\mathrm{D}}^{2}$ : l'interaction génotype $\times$ lieu est donc assez bien analysée par le modèle (1). Entre les lieux (Compiègne et Rennes), pour lesquels la différence $c_{j}-c_{j}^{\prime}$ est 1,17 , l'amplitude des écarts d'interaction est en moyenne de $1,17 \sigma_{\mathrm{D}}$ soit 7,6 $\mathrm{q} / \mathrm{ha}$.

La figure 2 peut suggérer que le génotype 37 a un poids particulier dans la définition de l'échelle $c_{j}$. Les estimations $c_{j}^{\prime}$ (tabl. 3) obtenues en supprimant ce point ne donnent cependant pas des lieux une classification radicalement différente. L'examen des teneurs en eau du grain à la récolte mène à penser que l'interaction analysée est du type précocité $\times$ disponibilité en sommes de température ou en rayonnement. Une analyse plus fine des conditions climatiques dans chaque lieu serait nécessaire.

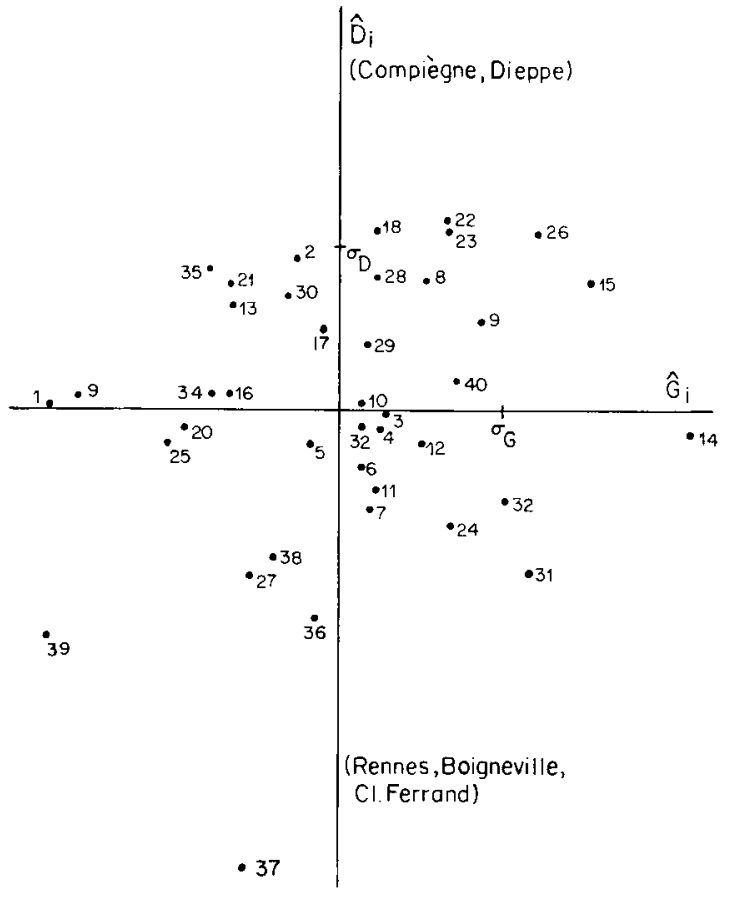

Figure 2

Représentation graphique des estimations du modèle (1).

Description of genotypes from the model (I).

Les coefficients de détermination génétique des variables $G_{i}$ et $D_{i}$ sont respectivement de 0,82 et de 0,69 : en d'autres termes, on apprécie mieux l'adaptation générale que l'adaptation spécifique, et ce d'autant plus que la corrélation $\left(\mathrm{G}_{\mathrm{i}}, \mathrm{D}_{\mathrm{i}}\right)$ est faible $(0,127)$.

Certains lieux, parce que leur $c_{j}$ est faible en valeur absolue, permettent une bonne expression de la valeur moyenne $\mathrm{G}_{i}$ : ce sont la Minière, Verneuil-l'Etang et Chateaudun. La méthode exposée dans un article précédent (VINCOURT \& GALLAIS, 1983) appliquée aux mêmes données, aboutissait à des résultats très voisins : les gains attendus en sélectionnant dans ces lieux étaient à la fois importants en moyenne et relativement stables d'un lieu à l'autre. Ce résultat ne fait d'ailleurs que confirmer la cohérence des 2 approches.

Remarque : A partir des paramètres du modèle (1), on pourrait également mesurer :
- le pouvoir de prédiction du lieu $\mathrm{j}_{0}$, par le coefficient de détermination génétique de la valeur générale $\mathrm{G}_{\mathrm{i}}+\gamma \mathrm{D}_{\mathrm{i}}$ à partir du prédicteur $\hat{\mathrm{G}}_{\mathrm{i}}+\mathrm{C}_{\mathrm{j}_{0}} \hat{\mathrm{D}}_{\mathrm{i}} ;$

- le pouvoir de révélation du lieu $\mathrm{j}_{0}$ par le coefficient de détermination de la valeur vraie $G_{i}+C_{j_{0}} D_{i}$ à partir du prédicteur $\hat{\mathrm{G}}_{\mathrm{i}}+\mathrm{C}_{\mathrm{j}_{\mathrm{o}}} \hat{\mathrm{D}}_{\mathrm{i}}$.

Ces critères n'ont bien sûr de sens que si le modèle (1) décrit correctement la situation, c'est-à-dire si $\sigma_{R}^{2}$ n'est pas trop grand.

\section{La situation à effets fixés}

Nous avons retenu pour tester cette méthode un essai de maïs grain conduit dans 6 lieux sur 9 combinaisons hybrides pour la station de Mons-en-Chaussée (M. DERIEUX, essai 21, 1979).

Le tableau 4 récapitule les effets des lieux estimés selon le modèle $2^{\prime}$. Le paramètre $m_{j}^{\prime}$ peut mesurer le pouvoir discriminant des lieux, et il semble que la précision intra-lieu, évaluée par $\hat{\sigma}_{\mathrm{j}}$, l'affecte de façon prépondérante. L'examen de $\mathrm{d}_{\mathrm{j}}$ permet d'appréhender quels lieux classent les variétés très différemment : à cet égard, le lieu Chateaulin, en situation océanique et fraîche, paraît se distinguer très nettement de tous les autres lieux.

TABLEAU 4

Caractérisation des lieux de tests dans le modèle $\left(2^{\prime}\right)$ (essai 21, Mons-en-Chaussée, 1979).

Description of the locations from the model (2').

Effets des lieux

\begin{tabular}{lccc} 
& $\mathrm{m}_{\mathrm{j}}$ & $\mathrm{c}_{\mathbf{j}}$ & $\sigma_{\mathrm{j}}$ \\
La Minière & 19,5 & 0,02 & 3,78 \\
Hargicourt & 21,4 & $-0,20$ & 2,70 \\
Mons-en-Chaussée & 28,7 & $-0,10$ & 2,29 \\
Wissembourg & 16,1 & $-0,32$ & 4,11 \\
Mirecourt & 26,5 & $-0,27$ & 2,79 \\
Chateaulin & 27,6 & 0,88 & 2,11 \\
\hline \hline
\end{tabular}

La représentation graphique (fig. 3) des effets variétaux $\mathrm{g}_{\mathrm{i}}^{\prime}$ et $\mathrm{d}_{\mathrm{i}}^{\prime}$ fait ressortir 2 points importants :

- le paramètre $\mathbf{g}_{\mathrm{i}}^{\prime}$ classe les variétés de la même façon que le critère « empirique » couramment utilisé, consistant à faire la moyenne des rapports à un témoin ;

- les variétés 5 et 6 , d'une part, $7,8,9$, d'autre part, sont en fait différentes versions cytoplasmiques de formules hybrides (HTV $246 \mathrm{~N}$ et C ; MB $125 \mathrm{~N}, \mathrm{C}$ et $\mathrm{T}$, respectivement). Le critère $\mathrm{g}_{\mathrm{i}}^{\prime}$ comme le critère empirique ne permettent pas de distinguer vraiment les 2 hybrides, alors que le critère $d_{i}^{\prime}$ les sépare très nettement. Autrement dit, c'est bien d'adaptation spécifique qu'il s'agit et le recours à cette méthode d'analyse a permis de mieux typer les comportements variétaux.

Il conviendrait bien sûr de mettre à l'épreuve cette technique sur un grand nombre de situations ; il nous semble cependant qu'elle permet à la fois d'enrichir l'information du sélectionneur et de mettre à sa disposition, en les fondant sur des bases un peu plus solides, des critères avec lesquels il a l'habitude de confronter son expérience (par exemple le coefficient de variation). 


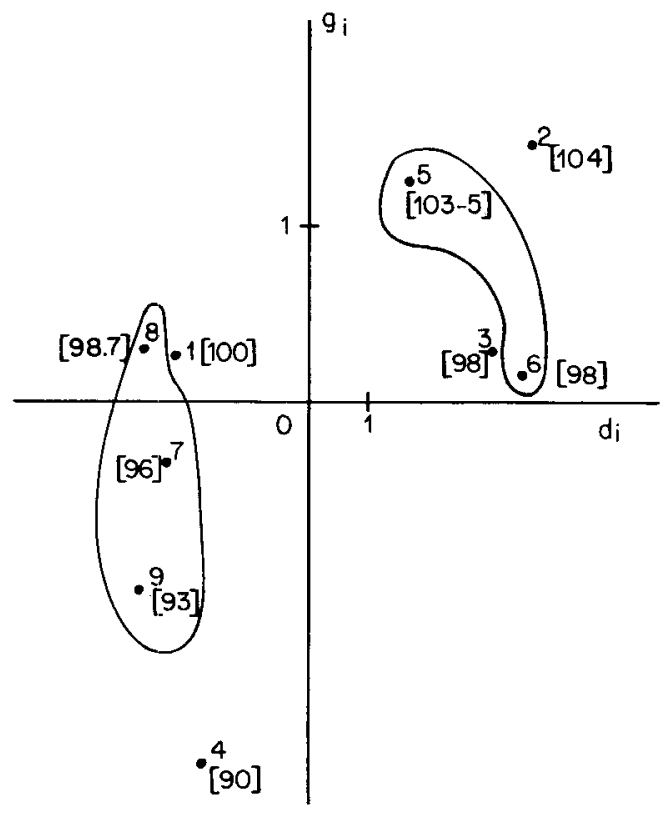

Figure 3

Caractérisation des génotypes dans le modèle (2').

$d_{i}$ et $g_{i}:$ parametre du modèle;

[]: critère empirique (voir le texte).

Description of genotypes from the model ( $\left.2^{\prime}\right)$.

[J : empirical criterion (see in text).

\section{CONCLUSION}

Nous avons rappelé, dans cet article, différentes techniques permettant d'utiliser avec profit l'information recueillie dans des essais multilocaux. Ces techniques ne sont pas originales dans le sens où leurs fondements théoriques ont fait l'objet de publications déjà anciennes (en particulier MANDEL, 1971). Toutefois, leur usage est très peu répandu parmi les sélectionneurs, alors que le besoin de valoriser une information coûteuse se fait davantage sentir. Nous voyons 2 avantages essentiels à leur emploi :

- bien que s'appliquant à des situations différentes, elles présentent une certaine cohérence dans la mesure où l'interaction génotype $\times$ lieu peut être appréhendée d'une façon plus synthétique ;
- elles permettent d'établir une certaine fusion entre les critères exhibés par l'analyse de variance classique, les concepts utilisés en sélection (coefficient de détermination, réponse à la sélection) et les critères empiriques qui aident aujourd'hui à la décision.

Les possibilités de calcul offertes désormais devraient permettre de les mettre à l'épreuve rapidement. Les programmes MULTILOC et HENDER écrits en FORTRAN (sur Mini 6, CII-HB) répondent à cet objectif et son actuellement disponibles.

Reçu le 16 mai 1983. Accepté le 15 mai 1984.

ANNEXE : Estimation des paramètres du modèle (2) (cf. II, A, 2)

- Soit $X$ la matrice $J \times J$ dont l'élément $x_{u v}$ est :

$$
\mathrm{x}_{\mathrm{uv}}=\frac{1}{\mathrm{I}-1} \sum_{\mathrm{i}}\left(\mathrm{Y}_{\mathrm{iu} .}-\mathrm{Y}_{\text {.u. }}\right)\left(\mathrm{Y}_{\mathrm{iv} .}-\mathrm{Y}_{. \mathrm{v} .}\right)-\delta_{\mathrm{uv}} \frac{\mathrm{u}^{\hat{\sigma}^{2}} \mathrm{E}}{\mathrm{L}_{\mathrm{u}}}
$$

avec $\delta_{\mathrm{uv}}=1$ si u $=\mathrm{v}, 0$ sinon

$\hat{\sigma}_{\mathrm{uE}}^{2}=$ estimation de la variance résiduelle dans le lieu $\mathrm{u}$.

Sous le modèle (2), l'espérance de $\mathrm{x}_{\mathrm{uv}}$ est :

(a) $\mathrm{E}\left(\mathrm{x}_{\mathrm{uv}}\right)=\sigma_{\mathrm{G}}^{2}+\mathrm{c}_{\mathrm{u}} \mathrm{c}_{\mathrm{v}} \sigma_{\mathrm{D}}^{2}+\rho \sigma_{\mathrm{G}} \sigma_{\mathrm{D}}\left(\mathrm{c}_{\mathrm{u}}+\mathrm{c}_{\mathrm{v}}\right)+\sigma_{\mathrm{R}}^{2} \delta_{\mathrm{uv}}$

La matrice $\mathrm{X}$ s'obtient à partir de la matrice de covariance entre les moyennes phénotypiques obtenues dans les différents lieux en soustrayant, dans la diagonale, les variances résiduelles intra-lieu attachées à ces moyennes.

- On pose $\tilde{x}_{u v}=x_{u v}-x_{u}-x_{. v}+x_{\text {... L'espérance, sous le }}$ modèle (1), de la matrice $\tilde{x}$ d'élément $\tilde{x}_{u v}$, admet pour plus grande valeur propre $\sigma_{\mathbf{D}}^{2}+\sigma_{\mathbf{R}}^{2}$ et le vecteur propre associé est $c=\left(c_{1}, \ldots c_{j}\right)$. On estime donc c comme lé vecteur propre associé à la plus grande valeur propre $\mu$ de $\tilde{\mathrm{x}}$.

- L'équation (a) et le mode d'estimation de c proposé ci-dessus permettent d'estimer $\sigma_{\mathrm{G}}^{2}, \sigma_{\mathrm{D}}^{2}, \rho, \sigma_{\mathrm{R}}^{2}$ à partir du système :

$$
\left\{\begin{aligned}
\mu & =\sigma_{\mathrm{D}}^{2}+\sigma_{\mathrm{R}}^{2} \\
\sum_{\mathrm{u}} \mathrm{x}_{\mathrm{uu}} & =\mathrm{J} \sigma_{\mathrm{G}}^{2}+\sigma_{\mathrm{D}}^{2}+\mathrm{J} \sigma_{\mathrm{R}}^{2} \\
\frac{1}{\mathrm{~J}} \sum_{\mathrm{u}, \mathrm{v}} \mathrm{x}_{\mathrm{uv}} & =\mathrm{J} \sigma_{\mathrm{G}}^{2}+\sigma_{\mathrm{R}}^{2} \\
\frac{1}{\mathrm{j}} \sum_{\mathrm{u}, \mathrm{v}} \mathrm{c}_{\mathrm{u}} \mathrm{x}_{\mathrm{uv}} & =\rho \sigma_{\mathrm{G}} \sigma_{\mathrm{D}}
\end{aligned}\right.
$$

\section{RÉFÉRENCES BIBLIOGRAPHIQUES}

Burdon R. D., 1979. Generalization of multi-trait selection indices using information from several sites. N.Z.J. For., 9, 2, 145-152.

Denis J. B., 1979. Structuration de l'interaction. Biom. Praxim., 19, 49-58.

Denis J. B., 1980. Analyse de régression factorielle. Biom. Praxim., 20, 1-34.

Denis J. B., Vincourt P., 1982. Panorama des méthodes statistiques d'analyse des interactions génotype $\times$ milieu. Agronomie, 2 (3), 219-230.

Eberhart S. A., Russell W. A., 1966. Stability parameters for comparing varieties. Crop Sci., 6, 36-40.

Falconer D. S., 1960. « Introduction to quantitative genetics ». Oliver \& Boyd (Edinburgh) Ed., 365 p.

Finlay K. W., Wilkinson G. N., 1963. The analysis of adaptation in plant breeding program. Aust. J. Agric. Res., 14, 742-754.

Gallais A., Vincourt P., Bertholleau J. C., 1983. Critères de sélection du maïs ensilage : corrélations génétiques et héritabilité. Agronomie, 3 (8), 751-760.
Goffinet B., Vincourt P., 1980. Prise en compte de l'interaction génotype $\times$ milieu en sélection végétale. In «Biométrie et Génétique », J. M. Legay et al., éd., p. 90-97.

Hardwick R. C., 1981. The analysis of genotype $\times$ environment interactions : what does it mean if varietal stability is linearly related to varietal performance. Euphytica, 30, 217-221.

Mallard J., Masson J. P., Douaire M., 1983. Interactions genotype $\times$ milieu et modèle mixte. I. - Modélisation. Genet. Sel. Evol., 15 (3), 379-394.

Mandel J., 1971. A new analysis of variance model for non-additive data. Technimetrics, 13, 1-18.

Scheffe H., 1959. The analysis of variance. Ed. J. WILLEY, New York, $477 \mathrm{p}$.

Utz H. F., 1972. Die Zerlegung der Genotyp $\times$ UmweltInteraktionen. EDV in Medizin und Biologie, 2, 52-59.

Vincourt P., Gallais A., 1983. Sur la recherche de critères de sélection : la régression géno-phénotypique. Agronomie, 3 (9), 827-830.

Yates F., Cochran W. G., 1938. The analysis of groups of experiments. J. Agric. Sci. Camb., 28, 556-580. 